\title{
Using the Edmonton obesity staging system to predict mortality in a population-representative cohort of people with overweight and obesity
}

\author{
Raj S. Padwal MSc MD, Nicholas M. Pajewski PhD, David B. Allison PhD, Arya M. Sharma MD PhD
}

\begin{abstract}
- Abstract
Background: Anthropometric-based classification schemes for excess adiposity do not include direct assessment of obesity-related comorbidity and functional status and thus have limited clinical utility. We examined the ability of the Edmonton obesity staging system, a 5-point ordinal classification system that considers comorbidity and functional status, in predicting mortality in a nationally representative US sample.
\end{abstract}

Methods: We analyzed data from the National Health and Human Nutrition Examination Surveys (NHANES) III (1988-1994) and the NHANES 1999-2004, with mortality follow-up through to the end of 2006. Adults (age $\geq 20 \mathrm{yr}$ ) with overweight or obesity who had been randomized to the morning session at the mobile examination centre were scored according to the Edmonton obesity staging system. We examined the relationship between staging system scores and mortality, and Cox proportional hazards models were adjusted for the presence of the metabolic syndrome or hypertriglyceridemic waist.

Results: Over $75 \%$ of the cohort with overweight or obesity were given scores of 1 or 2 .
Scores of 4 could not be reliably assigned because specific data elements were lacking. Survival curves clearly diverged when stratified by scores of $0-3$, but not when stratified by obesity class alone. Within the data from the NHANES 1988-1994, scores of 2 (hazard ratio $[\mathrm{HR}] 1.57$; $95 \%$ confidence interval $[\mathrm{Cl}]$ 1.16 to 2.13 ) and 3 (HR 2.69; $95 \% \mathrm{Cl} 1.98$ to 3.67) were associated with increased mortality compared with scores of 0 or 1 , even after adjustment for body mass index and the metabolic syndrome. We found similar results after adjusting for hypertriglyceridemic waist (i.e., waist circumference $\geq 90 \mathrm{~cm}$ and a triglyceride level $\geq 2 \mathrm{mmol} / \mathrm{L}$ for men; the corresponding values for women were $\geq 85 \mathrm{~cm}$ and $\geq 1.5 \mathrm{mmol} / \mathrm{L}$ ), as well as in a cohort eligible for bariatric surgery.

Interpretation: The Edmonton obesity staging system independently predicted increased mortality even after adjustment for contemporary methods of classifying adiposity. The Edmonton obesity staging system may offer improved clinical utility in assessing obesityrelated risk and prioritizing treatment.
$\mathrm{B}$ ody mass index (BMI) is the most common measure used to classify excess adiposity. A BMI of $18.5-24.9 \mathrm{~kg} / \mathrm{m}^{2}$ is considered normal, a BMI of $25.0-29.9 \mathrm{~kg} / \mathrm{m}^{2}$ is considered overweight and a BMI of more than $30 \mathrm{~kg} / \mathrm{m}^{2}$ is considered obese. ${ }^{1}$ Observational studies have consistently reported deleterious associations between elevated BMI and morbidity/mortality. ${ }^{2}$ Each five-unit increment in BMI above $25 \mathrm{~kg} / \mathrm{m}^{2}$ is associated with increases of $29 \%$ for overall mortality, $41 \%$ for vascular mortality and $210 \%$ for diabetesrelated mortality. ${ }^{3}$ Measures of central adiposity, including increased waist circumference, predict cardiometabolic risk independent of elevated BMI. ${ }^{4}$ Thus, measuring both BMI and central adiposity to classify and quantify obesity-related risk and assess the appropriateness of treatments such as starting the use of antiobesity drugs or bariatric surgery is recommended..$^{1,5}$

BMI is a useful population-based tool to classify adiposity and estimate its prevalence. ${ }^{6}$ However, BMI possesses well-known limitations at the individual level, ${ }^{7}$ including the inability to directly distinguish between lean and fat tissue. Thus, at a given BMI, substantial variation in adiposity can occur. ${ }^{8}$ Furthermore, neither BMI nor waist circumference directly reflects the presence of underlying obesityrelated comorbidity, reduced quality of life or diminished functional status - elements that are widely considered to be critically important to the clinical assessment of patients with
Competing interests: Raj Padwal and Arya Sharma are supported by an alternative funding plan from the Government of Alberta and the University of Alberta. David Allison has received grants, honoraria, donations, and consulting fees from numerous other commercial and nonprofit entities with interests in obesity. No other competing interests were declared.

This article has been peer reviewed.

Correspondence to: Dr. Raj S. Padwal, rpadwal@ualberta.ca

CMAJ 2011. DOI:10.1503 /cmaj.110387 
excess body weight. For example, BMI thresholds are currently used to determine eligibility for bariatric surgery. This approach has been criticized, and recommendations for scoring systems that incorporate assessments of comorbidity have been proposed as alternatives. ${ }^{9}$

Recently, we proposed a new clinical staging system that ranks people with excess adiposity on a 5-point ordinal scale, while incorporating obesity-related comorbidities and functional status into the assessment (Box 1). ${ }^{6}$ The Edmonton obesity staging system is intended to complement anthropometric measures, but it requires further validation. In this study, we examine the population distribution of Edmonton obesity staging system scores and assess the system's ability to predict mortality independent of anthropometric indices in a large, nationally representative US sample.

\section{Methods}

\section{Data collection}

We used data from the National Health and Human Nutrition Examination Surveys (NHANES), including the NHANES III (19881994) and the combination of three two-year cycles of the NHANES continuous surveys (1999-2004). ${ }^{10}$ The NHANES uses a complex, stratified, multistage probability cluster design to obtain nationally representative samples of the US civilian population, with the exception of those residing in institutions. Information on mortality from public-use mortality files was linked to the National Death Index, with followup through Dec. 31, 2006. Nonpregnant people with overweight or obesity aged 20 years or more with no history of cancer and who had been randomized to the morning session of the mobile

\section{Box 1: The Edmonton obesity staging system}

0 No apparent risk factors (e.g., blood pressure, serum lipid and fasting glucose levels within normal range), physical symptoms, psychopathology, functional limitations and/or impairment of well-being related to obesity

1 Presence of obesity-related subclinical risk factors (e.g., borderline hypertension, impaired fasting glucose levels, elevated levels of liver enzymes), mild physical symptoms (e.g. dyspnea on moderate exertion, occasional aches and pains, fatigue), mild psychopathology, mild functional limitations and/or mild impairment of well-being

2 Presence of established obesity-related chronic disease (e.g., hypertension, type 2 diabetes, sleep apnea, osteoarthritis), moderate limitations in activities of daily living and/or well-being

3 Established end-organ damage such as myocardial infarction, heart failure, stroke, significant psychopathology, significant functional limitations and/or impairment of well-being

4 Severe (potentially end-stage) disabilities from obesity-related chronic diseases, severe disabling psychopathology, severe functional limitations and/or severe impairment of well-being examination centre were considered eligible. Inclusion was limited to people from the morning session because fasting glucose measurements were needed to assign a score using the Edmonton obesity staging system. In total, 4373 respondents from the NHANES III and 3770 respondents from the NHANES 1999-2004 met our eligibility requirements. People for whom mortality information was missing or for whom a score could not be assigned were excluded $(n=6$ NHANES III respondents and $n=170$ NHANES 1999-2004 respondents). Final unweighted sample sizes were 4367 NHANES III respondents and 3600 NHANES 1999-2004 respondents.

Race/ethnicity was categorized into nonHispanic white, non-Hispanic black, MexicanAmerican and other. Smoking status was defined as current smoker, former smoker (lifetime exposure $\geq$ 100 cigarettes) or never smoked. When a respondent reported consuming alcohol, average annual alcohol consumption was calculated by multiplying the number of beverages ingested daily by the number of days on which alcohol was ingested.

The mobile examination centre consisted of four large trailers containing the equipment and personnel needed to perform physical and biochemical evaluations. Trained examiners used standardized protocols ${ }^{10}$ to measure weight, standing height and waist circumference. Excess adiposity was classified according to BMI into overweight $\left(25.0-29.9 \mathrm{~kg} / \mathrm{m}^{2}\right)$ and obese $\left(\geq 30 \mathrm{~kg} / \mathrm{m}^{2}\right.$ ) categories. Obesity was further subdivided into grades I (30-34.9 kg/m²), II (35.0$\left.39.9 \mathrm{~kg} / \mathrm{m}^{2}\right)$ and III $\left(\geq 40 \mathrm{~kg} / \mathrm{m}^{2}\right) .{ }^{1}$

The Institutional Review Board of the University of Alabama at Birmingham granted nonhuman research designation status for this study.

\section{Assigning Edmonton obesity staging system scores}

An Edmonton obesity staging system score was assigned to each person with a BMI of $25 \mathrm{~kg} / \mathrm{m}^{2}$ or more using data from the health interview, physical examination and laboratory investigations. Respondents with a self-reported history of angina, heart disease, heart failure and cerebrovascular disease were given a score of 3 . If these comorbidities were absent, each of nine obesity-related comorbidity variables and one physical function variable (Appendix 1, available at www.cmaj.ca/lookup/suppl/doi:10.1503 /cmaj.110387/-/DC1) were scored. Each variable was scored separately, resulting in 10 different possible scores for each subject. Subsequently, the highest score across the 10 categories was assigned as the final Edmonton obesity staging system score for that patient. For example, a patient with diabetes (score 2), 
osteoarthritis (score 2), no functional limitation (score 0 ) and no other comorbidity would be assigned a score of 2 . Respondents for whom we had insufficient information to score at least 5 out of 10 subcategories were excluded. We had planned to examine psychological function and additional comorbidities (e.g., sleep apnea, obesity hypoventilation syndrome, gastroesophageal reflux disease) and to also include Edmonton obesity staging system scores of 4 in our analysis (Box 1). However, these variables could not be reliably assessed owing to insufficient data.

\section{Obesity-related comorbidities}

The obesity-related comorbidity variables were diabetes, hypertension, dyslipidemia, osteoarthritis, liver disease, kidney disease, limitations to physical functioning, metabolic syndrome and hypertriglyceridemic waist. The presence of each was determined using the definitions in Appendix 1.

\section{Eligibility for bariatric surgery}

The predictive ability of the Edmonton obesity staging system independent of BMI and meta-

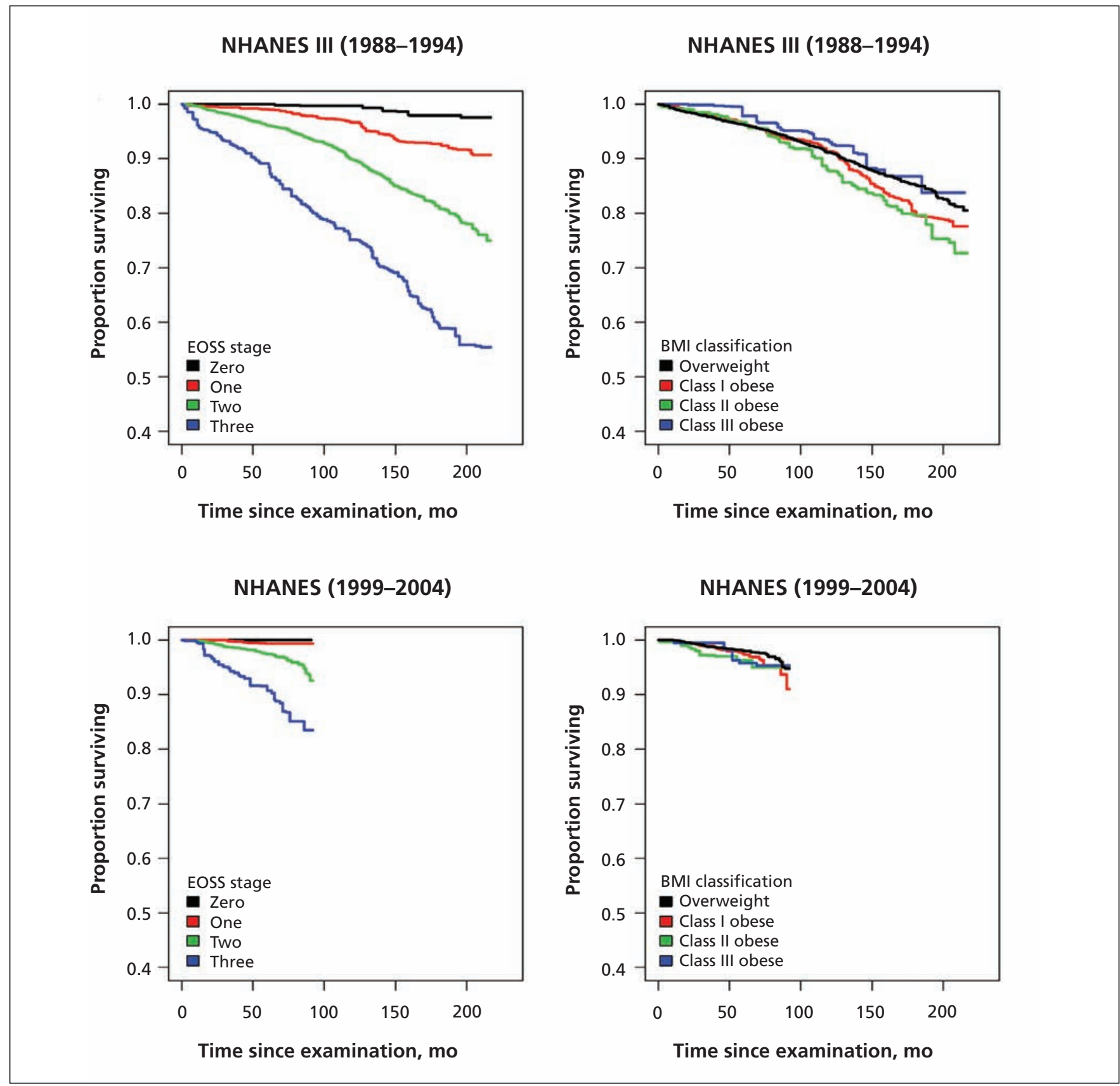

Figure 1: Comparison of staging system and anthropometric classification scheme for predicting all-cause mortality among people with overweight and obesity. BMI = body mass index, EOSS = Edmonton obesity staging system, NHANES = National Health and Human Nutrition Examination Surveys. 
bolic syndrome or hypertriglyeridemic waist was also examined in a subgroup of people eligible for bariatric surgery. Eligibility was defined according to current guidelines and expert consensus. ${ }^{1,5,11}$ Criteria included age 20-64 years, a BMI of $40 \mathrm{~kg} / \mathrm{m}^{2}$ or more, or a BMI of 35.0-39.9 $\mathrm{kg} / \mathrm{m}^{2}$ plus one or more of the following: fasting glucose of $7.0 \mathrm{mmol} / \mathrm{L}$ or more; systolic blood pressure higher than $140 \mathrm{~mm} \mathrm{Hg}$; diastolic blood pressure higher than $90 \mathrm{~mm} \mathrm{Hg}$; triglyceride level of more than $2.8 \mathrm{mmol} / \mathrm{L}$; total cholesterol level of more than $5.7 \mathrm{mmol} / \mathrm{L}$; high-density lipoprotein cholesterol level less than $0.9 \mathrm{mmol} / \mathrm{L}$; low-density lipoprotein cholesterol level higher than $5.2 \mathrm{mmol} / \mathrm{L}$; and self-reported current use of insulin, oral hypoglycemic agents or antihypertensive agents.

\section{Statistical methods}

We analyzed mortality using Kaplan-Meier survival curve estimates and Cox proportional hazards regression. Only unadjusted survival curves are reported for the data from the NHANES 1999-2004 owing to insufficient

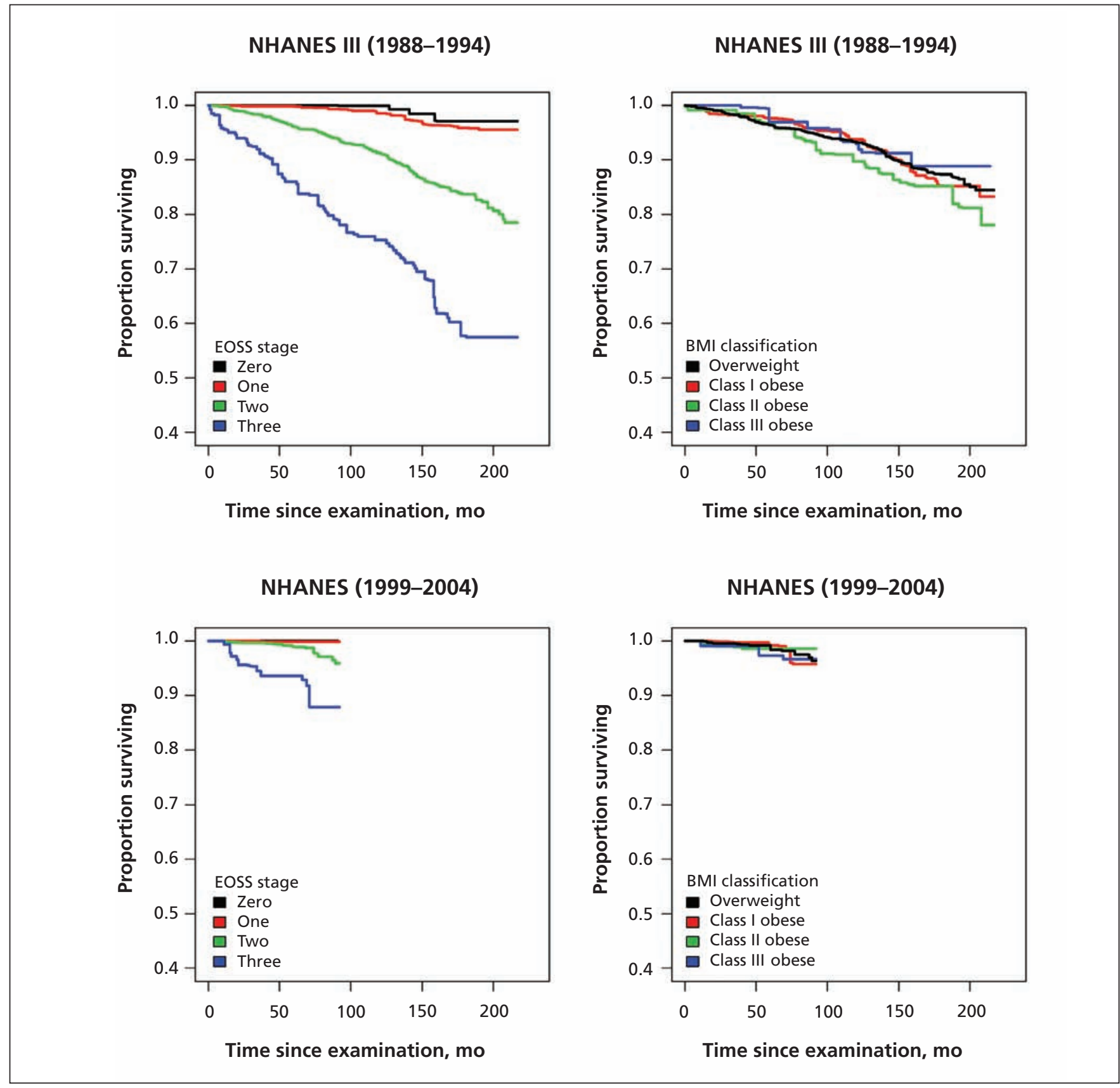

Figure 2: Subgroup analysis of people who had never smoked, comparing staging system and anthropometric classication scheme as predictors of all-cause mortality among people with overweight and obesity. BMI = body mass index, EOSS = Edmonton obesity staging system, NHANES = National Health and Human Nutrition Examination Surveys. 
power and limited follow-up. To eliminate the potentially confounding effect of smoking, we analyzed the subgroup of people who reported never having smoked.

Age was used as the time scale ${ }^{12}$ for the Cox models, also accounting for left truncation induced by variable age at baseline. No other adjustment for age was done. ${ }^{13}$ The proportional hazard assumption was evaluated using Schoenfeld residuals. ${ }^{14}$ Sex, BMI category (overweight and class I, II, or III obesity) and Edmonton obesity staging system score (0-3) were included as model predictors. We adjusted for the presence of the metabolic syndrome and hypertriglyceridemic waist in separate models. Owing to the evidence of deviation from the proportional hazards, separate baseline hazards were fit for each combination of race/ethnicity (white, black and other) and smoking status (current, former and never smoked). In addition, the effect of hypertriglyceridemic waist was modelled as a timedependant covariate with different coefficients for 60 years of age or younger, 61-82 years of age, and older than 82 years. These thresholds were based on the distribution of age ranges within the dataset.

All analyses accounted for the complex sampling design of the NHANES surveys. Analyses were performed using SAS version 9.1.3 (SAS Inc., Cary, NC) and the survey package $^{15}$ for the R Statistical Computing Environment. ${ }^{16}$

\section{Results}

Baseline characteristics of the respondents to the NHANES 1988-94 and NHANES 1999-2004 are presented in Appendix 2 (available at www .cmaj.ca/lookup/suppl/doi:10.1503/cmaj.110387 /-/DC1). Of the overweight or obese respondents in the NHANES 1988-94, 77.2\% received scores of 1 or 2 using the Edmonton obesity staging system; the corresponding rate for the NHANES 1999-2004 cohort was 90.3\% (Appendix 2). Of all respondents, $43.9 \%$ met the criteria for the metabolic syndrome and $38.2 \%$ met the criteria for hypertriglyceridemic waist in both surveys. About $13.8 \%$ of respondents met

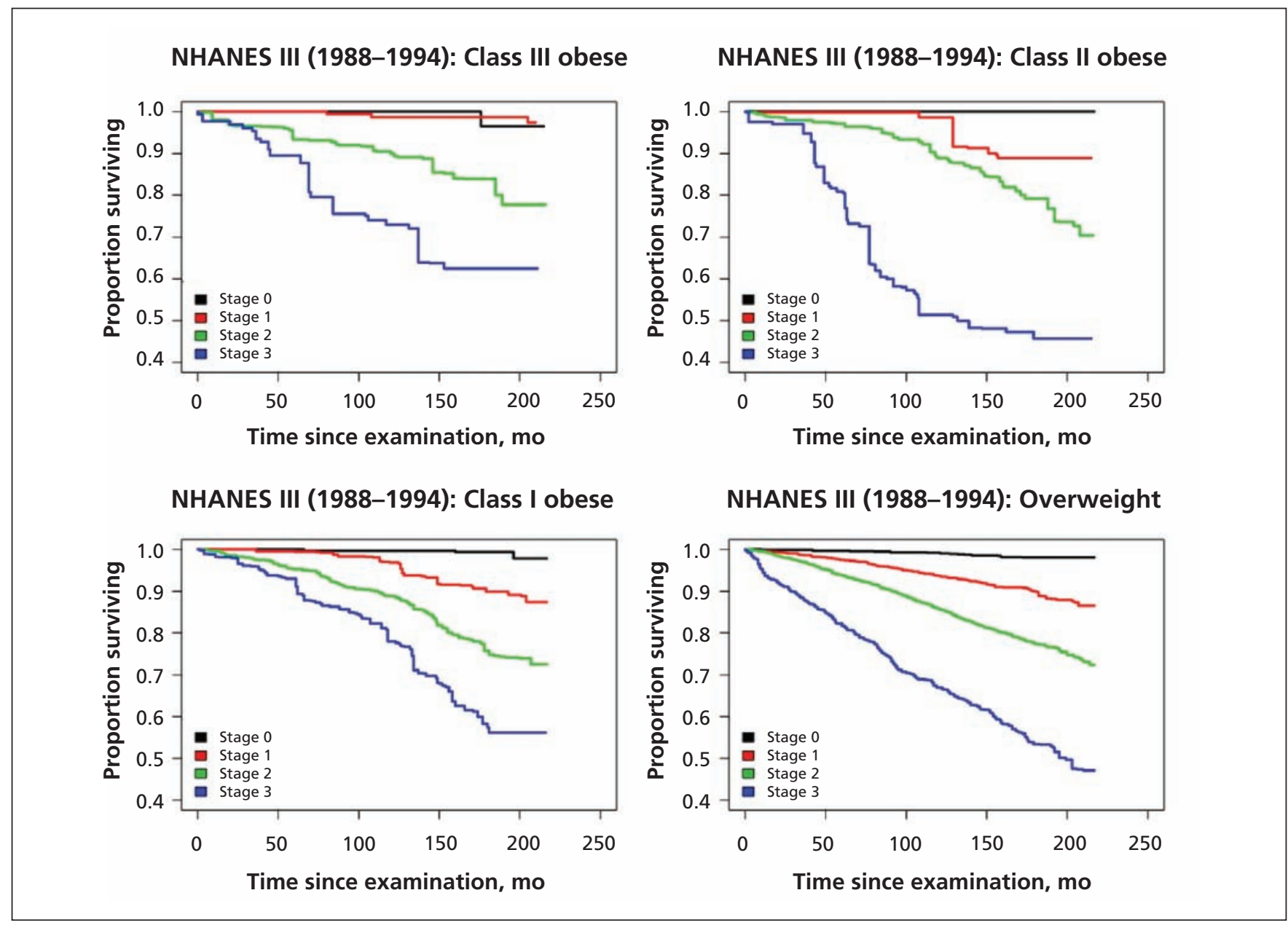

Figure 3: Comparison of the staging scheme's ability to predict all-cause mortality within BMI categories. BMI = body mass index, EOSS = Edmonton obesity staging system, NHANES = National Health and Human Nutrition Examination Surveys. 
Table 1: Adjusted Cox proportional hazard ratios showing the ability of the Edmonton obesity staging system to predict all-cause mortality in the overall population and within a subgroup of people eligible for bariatric surgery*

\begin{tabular}{|c|c|c|}
\hline & $\begin{array}{l}\text { Model adjusted for } \\
\text { presence of metabolic } \\
\text { syndrome, } \\
\text { HR }(95 \% \mathrm{Cl})\end{array}$ & $\begin{array}{l}\text { Model adjusted for } \\
\text { presence of } \\
\text { hypertriglyceridemic } \\
\text { waist, HR }(95 \% \mathrm{Cl})\end{array}$ \\
\hline \multicolumn{3}{|l|}{$\begin{array}{l}\text { Overall } \\
\text { population }\end{array}$} \\
\hline Female sex & $0.77(0.60-0.98)$ & $0.78(0.61-0.99)$ \\
\hline \multicolumn{3}{|l|}{$\begin{array}{l}\text { Edmonton obesity } \\
\text { staging system score }\end{array}$} \\
\hline $0 / 1$ & Reference & Reference \\
\hline 2 & $1.57(1.16-2.13)$ & $1.62(1.19-2.21)$ \\
\hline 3 & $2.69(1.98-3.67)$ & $2.78(2.07-3.74)$ \\
\hline \multicolumn{3}{|l|}{ BMI } \\
\hline Overweight & Reference & Reference \\
\hline Class I obesity & $1.22(1.01-1.48)$ & $1.26(1.03-1.55)$ \\
\hline Class II obesity & $1.73(1.23-2.46)$ & $1.81(1.27-2.57)$ \\
\hline Class III obesity & $1.52(0.94-2.46)$ & $1.61(1.02-2.56)$ \\
\hline $\begin{array}{l}\text { Presence of } \\
\text { metabolic syndrome }\end{array}$ & $1.14(0.98-1.33)$ & - \\
\hline \multicolumn{3}{|l|}{$\begin{array}{l}\text { Presence of } \\
\text { hypertriglyceridemic } \\
\text { waist, age, yr }\end{array}$} \\
\hline$\leq 60$ & - & $1.18(0.72-1.94)$ \\
\hline$>60, \leq 82$ & - & $0.90(0.71-1.14)$ \\
\hline$>82$ & - & $0.89(0.70-1.13)$ \\
\hline \multicolumn{3}{|l|}{$\begin{array}{l}\text { Subgroup eligible } \\
\text { for bariatric } \\
\text { surgery }\end{array}$} \\
\hline Female sex & $0.19(0.09-0.41)$ & $0.19(0.09-0.39)$ \\
\hline \multicolumn{3}{|l|}{$\begin{array}{l}\text { Edmonton obesity } \\
\text { staging system score }\end{array}$} \\
\hline $0 / 1$ & Reference & Reference \\
\hline 2 & $3.86(0.79-18.79)$ & $4.25(0.84-21.59)$ \\
\hline 3 & $12.29(2.10-72.00)$ & $13.50(2.28-80.00)$ \\
\hline \multicolumn{3}{|l|}{ BMI } \\
\hline Class II obesity & Reference & Reference \\
\hline Class III obesity & $0.94(0.44-2.00)$ & $0.93(0.42-2.10)$ \\
\hline $\begin{array}{l}\text { Presence of } \\
\text { metabolic syndrome }\end{array}$ & $1.33(0.58-3.08)$ & - \\
\hline \multicolumn{3}{|l|}{$\begin{array}{l}\text { Presence of } \\
\text { hypertriglyceridemic } \\
\text { waist, age, yr }\end{array}$} \\
\hline$\leq 60$ & - & $0.74(0.32-1.68)$ \\
\hline$>60$ & - & $1.04(0.40-2.69)$ \\
\hline \multicolumn{3}{|c|}{$\begin{array}{l}\text { Note: } \mathrm{BMI}=\text { body mass index, } \mathrm{Cl}=\text { confidence interval, } \mathrm{HR}=\text { hazard ratio. } \\
\text { *Data are from the National Health and Human Nutrition Examination Survey III (1988- } \\
\text { 1994). The model is stratified to allow for different baseline hazards according to all } \\
\text { combinations of race/ethnicity (white, black and other) and smoking status (current smoker, } \\
\text { former smoker or never smoked). } \\
\text { tHypertriglyceridemic waist was modeled by age as a time-dependent covariate owing to } \\
\text { evidence of deviation from proportional hazards when constant effects were assumed over } \\
\text { all attained ages. }\end{array}$} \\
\hline
\end{tabular}

the criteria for bariatric surgery. Across all BMI categories, most respondents received a score of 2 ; the next most common score was 1 (Appendix 3, available at www.cmaj.ca/lookup /suppl/doi:10.1503/cmaj.110387/-/DC1).

Kaplan-Meier plots examining mortality according to Edmonton obesity staging system score for the overall population, for the subgroup of people who had never smoked and within each BMI class showed marked differences between the survival curves for each obesity stage (Figures 1-3). In contrast, when mortality was assessed only according to BMI class, separation of the curves was far less apparent (Figures 1 and 2).

Using data from the NHANES 1988-94, multivariable Cox proportional hazards modelling showed that the adjusted hazard ratio (HR) for death increased with higher Edmonton obesity staging system scores (because of the small number of observed deaths for respondents with a score of 0 , scores 0 and 1 were collapsed into a single category). Scores of 2 (HR 1.57, 95\% CI 1.16-2.13) and 3 (HR 2.69, 95\% CI 1.983.67) were associated with a higher adjusted mortality hazard compared with scores of 0 or 1 even after adjustment for the metabolic syndrome (Table 1). Results were similar after adjusting for hypertriglyceridemic waist and in the cohort of respondents eligible for bariatric surgery (Table 1).

\section{Interpretation}

\section{Main findings}

Within a nationally representative cohort, higher Edmonton obesity staging system scores were a strong predictor of increasing mortality in both the overall population and in a cohort of people eligible for bariatric surgery. This ability was independent of BMI and the presence of metabolic syndrome or hypertriglyceridemic waist. Even within strata of BMI categories, there was clear separation of survival curves according to Edmonton obesity staging system scores.

The Edmonton obesity staging system does not directly or indirectly measure adiposity, therefore it cannot be used to define excess adiposity. Rather, the system is intended to complement anthropometric indices and provide incremental clinically relevant prognostic information in a manner analogous to the "tumor, node, metastasis" system widely used in oncology to define the size and extent of the spread of cancer. ${ }^{6}$ The major incremental contribution of this staging system to anthropometric indices and cardiovascular risk equations is the direct mea- 
surement of the presence and severity of underlying obesity-related comorbidities, which enables a more comprehensive and individualized assessment of risk.

Such enhanced risk assessment may enable a greater understanding of obesity-related prognosis and may also assist in determining the urgency of intervention. This may be particularly useful as a means for prioritizing patients for bariatric surgery. Surgery is widely considered to be an effective treatment for severe obesity. ${ }^{1}$ However, surgery is difficult to access in Canada because of high demand and limited capacity, which has led to the emergence of protracted, multiyear wait times. ${ }^{17,18}$ Currently, most patients are selected for surgery on the basis of BMI thresholds alone and have few obesityrelated comorbidities. The practice of selecting patients using BMI alone has been criticized as inaccurate and arbitrary and may result in the selection of patients who are least likely to benefit from weight reduction. ${ }^{9}$ Prioritizing patients with higher Edmonton obesity staging system scores - and thus greater comorbidity and risk of death - may help maximize the benefits of surgery. Further study, including full characterization of the risks and benefits, especially for patients with the highest scores, is required.

\section{Limitations}

Comorbidities within the Edmonton obesity staging system, such as diabetes and osteoarthritis, have been arbitrarily assigned to be equivalent in terms of their burden of illness. It is not yet clear whether certain comorbidities should receive a higher weighting.

Because this study was a retrospective analysis of pre-existing data, certain pertinent data for assigning Edmonton obesity staging system scores, such as respondents' psychological functioning, were unavailable. In addition, some comorbidities were based on self-report or were inferred as present based on the absence of other common causes (e.g., fatty liver disease), thus they may be subject to a higher risk of bias than direct measurements.

We have examined total mortality only; other end points such as quality of life and costs merit assessment.

No differentiation between types of diabetes could be made (of Americans with diabetes, 90\%-95\% have type 2 diabetes). ${ }^{19}$ However, both subtypes would require management in an overweight or obese cohort and were thus judged appropriate for inclusion.

A final potential limitation is that the most recent mortality follow-up available was from 2006. Although not contemporaneous, we feel that the results of this study remain relevant to people who currently have overweight or obesity. We do, however, acknowledge the need for additional follow-up analyses.

\section{Conclusion}

The Edmonton obesity staging system is a strong predictor of increasing mortality independent of BMI, metabolic syndrome and hypertriglyceridemic waist. We propose that this system be considered adjunctive to current anthropometric classification systems in assessing obesity-related risk, determining prognosis and guiding treatment. Further refinement of the staging system would assist in determining the best way of incorporating it into clinical care.

\section{References}

1. Lau DCW, Douketis JD, Morrison KM, et al. 2006 Canadian clinical practice guidelines on the management and prevention of obesity in adults and children. CMAJ 2007;176(Suppl 8): 1-117.

2. Hu FB. Obesity and mortality: watch your waist, not just your weight. Arch Intern Med 2007;167:875-6.

3. Prospective Studies Collaboration, Whitlock G, Lewington S, Sherliker P, et al. Body-mass index and cause-specific mortality in 900000 adults: collaborative analyses of 57 prospective studies. Lancet 2009;373:1083-96.

4. Yusuf S, Hawken S, Ounpuu S, et al. Unknown. Obesity and the risk of myocardial infarction in 27,000 participants from 52 countries: a case-control study. Lancet 2005;366:1640-9.

5. National Heart, Lung, and Blood Institute, National Institutes of Health. Clinical guidelines on the identification, evaluation, and treatment of overweight and obesity in adults: the evidence report. Bethesda (MD): National Institutes of Health. 1998.

6. Sharma AM, Kushner R. A proposed clinical staging system for obesity. Int J Obes (Lond) 2009;33:289-95.

7. Frankenfield DC, Rowe WA, Cooney RN, et al. Limits of body mass index to detect obesity and predict body composition. Nutrition 2001;17:26-30.

8. Gallagher D, Heymsfield SB, Heo M, et al. Healthy percentage body fat ranges: an approach for developing guidelines based on body mass index. Am J Clin Nutr 2000;72:694-701.

9. Pories WJ, Dohm L, Mansfield C. Beyond the BMI: the search for better guidelines for bariatric surgery. Obesity (Silver Spring) 2010;18:865-71.

10. Centers for Disease Control and Prevention (CDC). National Health and Nutrition Examination Survey Data. Hyattsville (MD): US Department of Health and Human Services, Centers for Disease Control and Prevention. Available: www.cdc.gov /nchs/nhanes.htm (accessed 2011 Aug. 5).

11. Yermilov I, McGory ML, Shekelle P, et al. Appropriateness criteria for bariatric surgery: beyond the NIH guidelines. Obesity (Silver Spring) 2009;17:1521-7.

12. Korn EL, Graubard BI, Midthune D. Time-to-event analysis of longitudinal follow-up of a survey: choice of the time-scale. Am J Epidemiol 1997; 145:72-80.

13. Gail MH, Graubard B, Williamson DF, et al. Comments on 'Choice of time scale and its effect on significance of predictors in longitudinal studies'. Stat Med 2009;28:1315-7.

14. Grambsch PM, Therneau TM. Proportional hazards tests and diagnostics based on weighted residuals. Biometrika 1994;81: 515-26.

15. Lumley T. Analysis of complex sample surveys. J Stat Softw 2004;9:1-19.

16. $R$ : a language and environment for statistical computing [computer program]. Vienna, Austria: R Foundation for Statistical Computing; 2009.

17. Flum DR, Khan TV, Dellinger EP. Toward the rational and equitable use of bariatric surgery. JAMA 2007;298:1442-4.

18. Padwal RS, Sharma AM. Treating severe obesity: morbid weights and morbid waits. CMAJ 2009;181:777-8.

19. National diabetes statistics, 2011. Bethesda (MD): National Diabetes Information Clearinghouse; 2011. Available: http ://diabetes.niddk.nih.gov/dm/pubs/statistics (accessed 2011 June 2). 
Affiliations: From the Department of Medicine (Padwal, Sharma), University of Alberta, Edmonton, Alta.; Section on Statistical Genetics (Pajewski, Allison), and the Nutrition Obesity Research Center (Allison), University of Alabama at Birmingham, Birmingham, Ala.; and the Department of Biostatistical Sciences (Pajewski), Wake Forest University Health Sciences, Winston-Salem, NC.

Contributors: Arya Sharma and Raj Padwal proposed the initial study idea, and all of the authors contributed to the development and further refinement of the project. Nicholas Pajewski had full access to all of the study data and takes responsibility for the integrity of the data and the accuracy of the data analysis. Raj Padwal and Nicholas Pajewski wrote the initial draft of the manuscript. Subsequent revisions con- tained input from all of the authors, and all of the authors approved the final version submitted for publication. No writing assistance was used in the production of this manuscript.

Funding: This research was supported in part by National Imstitutes of Health grant T32 HL072757. The sponsors had no role in the design and conduct of the study; collection, management, analysis, and interpretation of the data; and preparation, review, or approval of the manuscript.

Acknowledgements: The authors are grateful to Dr. Renee Desmond from the University of Alabama at Birmingham for her advice on some statistical aspects. The abstract of the article was presented at the National Obesity Conference in Montréal, Quebec, May 2011. 